\title{
Efficient transmission of IncFIlY and IncL plasmids and Klebsiella pneumoniae ST101 clone producing OXA-48, NDM-1 or OXA-181 in Bucharest hospitals
}

Sir,

Carbapenemase-producing Enterobacteriaceae are increasingly reported, with NDM1 (class B) and OXA-48 (class D) being the most widespread enzymes worldwide [1]. In Romania, a country with a high rate of carbapenemase-producing enterobacterial isolates (http://ecdc.europa.eu/en/healthtopics/antimicrobial_resistance/database/Pages/data base.aspx), molecular epidemiological data are scarce and are limited to certain regions of the country [2]. We aimed to elucidate the epidemiology and to provide detailed molecular data (genetic platforms, plasmid types and clones) for carbapenemase-producing Enterobacteriaceae from two hospitals in Bucharest, Romania.

A total of 21 carbapenemase-producing Enterobacteriaceae isolates (14 Klebsiella pneumoniae, 6 Enterobacter cloacae complex and 1 Escherichia coli) recovered from patients hospitalised in different units of two central hospitals between MarchDecember 2012 were characterised (Table 1). They corresponded to all carbapenemase-producing isolates identified among multidrug-resistant Enterobacteriaceae resistant to ertapenem [according to Clinical and Laboratory Standards Institute (CLSI) 2012 guidelines]. 
Identification of isolates and antibiotic susceptibility testing were performed by automated (VITEK II, MicroScan) and standard methods ( $h s p 60$ genotyping for $E$. cloacae, disk diffusion) (http://www.eucast.org). Carbapenemase production and

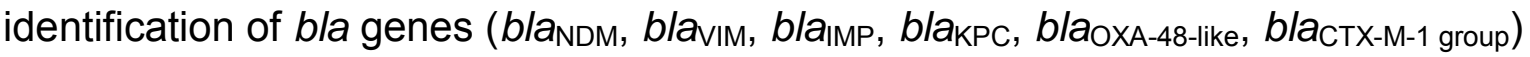
were performed as described previously [3] and the genetic context of bla $a_{\mathrm{NDM}-1}$, blaoxA-48 and blaoxA-181 was determined by PCR and sequencing (Supplementary Table S1). The relationship among the isolates was established by pulsed-field gel electrophoresis (PFGE) and multilocus sequence typing (MLST) for E. coli (http://mlst.warwick.ac.uk/mlst/) as well as for representative K. pneumoniae (http://bigsdb.pasteur.fr/klebsiella/klebsiella.html) and Enterobacter hormaechei (http://pubmlst.org/ecloacae) strains. Conjugation experiments were performed on isolates representing variable clones or plasmids by broth/solid mating as described previously [3]. Plasmid content was characterised by PCR-based replicon typing (PBRT) and replicon sequence typing (RST) (http://pubmlst.org/plasmid/), S1 nuclease PFGE and hybridisation (bla NDM-1 $_{1}$, bla $a_{\mathrm{OXA}-48}$, bla $\mathrm{OXX}_{\mathrm{O}-181}$, bla $\mathrm{a}_{\mathrm{CTX}-\mathrm{M}-15}$, rep probes) as reported previously [3]. Conserved regions of the prototype pOXA-48 plasmid (repA, traU, parA; GenBank accession no. JN626286) were characterised by PCR and sequencing for representative OXA-48- and OXA-181-producing strains, and relatedness among OXA-48-encoding plasmids was evaluated by restriction fragment length polymorphism (RFLP) using EcoRI as restriction enzyme [4].

Eight isolates of different Enterobacteriaceae produced NDM-1 (six E. hormaechei, one K. pneumoniae and one E. coli), eleven K. pneumoniae produced OXA-48 and two K. pneumoniae produced both NDM-1 and OXA-181 (differing from OXA-48 in four amino acid changes, whilst the corresponding bla genes are $94 \%$ homologous), 
and most of them encoded also CTX-M-15 (Table 1). These data, together with previous reports, support that the Balkan region might be an important reservoir of these carbapenemases [2].

NDM-1-producers were assigned to the worldwide spread ST74 or ST171 E. hormaechei (http://pubmlst.org/ecloacae) or ST101 K. pneumoniae clones and the apparently sporadic ST5 E. coli clone (http://mlst.warwick.ac.uk/mlst/). The bla $a_{\mathrm{NDM}-1}$ gene in all bacterial species was located within conjugative $100 \mathrm{~kb}$ IncFll plasmids (Y4:A-:B42) containing a genetic platform (ISCR 14/6-rmtC- $\Delta$ ISAba125-bla NDM-1- $^{-}$ ble $\left.e_{\mathrm{MBL}-t} \operatorname{tr} F-g r o E L\right)$ identical to those identified in different Enterobacteriaceae species (K. pneumoniae, Klebsiella oxytoca, E. cloacae), suggesting efficient transmission in the nosocomial setting [5]. When detected, blacTX-M-15 was located within IncR plasmids.

Isolates producing NDM-1 and OXA-181 also belonged to identical ST101 K. pneumoniae. The bla $a_{\mathrm{NDM}-1}$ gene was chromosomally located embedded in an identical genetic platform to that described above (ISCR 14/6-rmtC-AISAba125bla $\left.a_{\mathrm{NDM}-1}-b / e_{\mathrm{MBL}-} \operatorname{trp} F-g r o E L\right)$, suggesting a common origin. ISEcp1 was found upstream of the bla OXA-181 gene and also upstream of bla carrying $\operatorname{rep} A$, traU and parAgenes identical to those from pOXA-48-like plasmids [4]. Thus, we report for the first time the acquisition of ISEcp 1-blaoXA-181 by a pOXA48-like IncL plasmid, instead of previously described locations (7.6 kb ColE, GenBank accession no. JN205800; 84 kb IncT, GenBank accession no. JQ996150; $60 \mathrm{~kb}$ IncN, GenBank accession no. $\underline{\mathrm{KM} 660724)}$. $\mathrm{FII}_{\mathrm{K} 1}$ and $\mathrm{FII}_{2}$ were also detected in these isolates. 
Most OXA-48-producing isolates belonged to K. pneumoniae ST101 and sporadically to ST395 K. pneumoniae, both sequence types commonly linked to the intercontinental dissemination of blaOXA-48 and previously described in Romania $[1,2]$. We observed that in the region analysed, blaoxA-48 was located in a Tn1999.2 transposon variant (upstream IS 1999 sequence disrupted by IS1) within closely related 50-60 kb pOXA-48-like conjugative IncL plasmids, which constitute the most common vehicles for bla OXA-48 (Tn1999.2, IncL) described worldwide [1,4]. These isolates simultaneously carried IncR (where bla replicons (Table 1). The association of ST101 K. pneumoniae with different carbapenemases suggests extended dissemination of this clone in Bucharest and elsewhere in Romania [2], and the differences in the plasmidome of isolates producing NDM-1 (IncFII, IncR), NDM-1 and OXA-181 (IncL, IncFII 1 , IncFII 2 ) or OXA-48 (IncL, IncFII 2 , IncR) enzymes (Table 1) highlights its ability to acquire variable genetic platforms.

This study provides a detailed characterisation of carbapenemase-producing Enterobacteriaceae from Bucharest (Romania) at the molecular level (genetic platforms, plasmids, clones) and additional evidence that the Balkan region constitutes an important reservoir of NDM-1, OXA-48 and OXA-181 carbapenemases. These data highlighted the efficient nosocomial transmission of specific plasmid backbones between different Enterobacteriaceae species (IncFII carrying b/a $a_{\mathrm{NDM}-1}$ ) or within K. pneumoniae ST101 lineage (b/aOXA-48 $/$ bla OXA-181 $_{\text {and }}$ IncL), which was the predominant lineage involved in the dissemination of different carbapenemases in this geographic region. 
Acknowledgments: The authors thank Otilia Banu and Elvira Borcan from the clinical institutions studied for providing the bacterial strains and for their automatic identification and antibiotic susceptibility testing.

Funding: This work received financial support from the European Union (FEDER funds) through Programa Operacional 'Factores de Competitividade' - COMPETE and from national funds [Fundação para a Ciência e Tecnologia (FCT)] through grants UID/Multi/04378/2013 and NORTE-07-0124-FEDER-000066 (under the framework of QREN). IC was supported by Ideas 1716/2011 grant [contract no. 154/05.10.2011]. ÂN and CR were supported by FCT grants [references SFRH/BPD/104927/2014 and SFRH/BD/84341/2012, respectively]. The funding sources had no involvement in the study design, execution or article preparation.

Competing interests: None declared.

Ethical approval: Not required.

\section{References}

[1] Potron A, Poirel L, Rondinaud E, Nordmann P. Intercontinental spread of OXA-48 $\beta$-lactamase-producing Enterobacteriaceae over a 11-year period, 2001 to 2011. Euro Surveill 2013;18:pii: 20549.

[2] Dortet L, Flonta M, Boudehen YM, Creton E, Bernabeu S, Vogel A, et al. Dissemination of carbapenemase-producing Enterobacteriaceae and 
Pseudomonas aeruginosa in Romania. Antimicrob Agents Chemother 2015;59:7100-3.

[3] Rodrigues C, Novais Â, Machado E, Peixe L. Detection of VIM-34, a novel VIM-1 variant identified in the intercontinental ST15 Klebsiella pneumoniae clone. J Antimicrob Chemother 2014;69:274-5.

[4] Carattoli A, Seiffert SN, Schwendener S, Perreten V, Endimiani A. Differentiation of IncL and IncM plasmids associated with the spread of clinically relevant antimicrobial resistance. PLoS One 2015;10:e0123063.

[5] Wu W, Feng Y, Carattoli A, Zong Z. Characterization of an Enterobacter cloacae strain producing both KPC and NDM carbapenemases by wholegenome sequencing. Antimicrob Agents Chemother 2015;59:6625-8.

Ilda Czobor ${ }^{a, b, 1,2}$ Ângela Novais ${ }^{a, 1}$ Carla Rodrigues $^{a}$ Mariana Carmen Chifiriuc ${ }^{\text {b,c }}$ Grigore Mihăescu b Veronica Lazăr ${ }^{\text {b }}$ Luísa Peixe $^{\mathrm{a}, *}$

a UCIBIO/REQUIMTE, Faculdade de Farmácia, Universidade do Porto, Porto, Portugal ${ }^{\mathrm{b}}$ University of Bucharest, Faculty of Biology, Department of Microbiology, Portocalelor no. 1-3, district 6, Bucharest, Romania ${ }^{\mathrm{C}}$ Institute of Research of University of Bucharest, ICUB, Splaiul Independent ei 9195, district 5, Bucharest, Romania 
* Corresponding author. Present address: REQUIMTE Research Group, Microbiology Laboratory, Faculty of Pharmacy, University of Porto, Rua Jorge Viterbo Ferreira n² 228, 4050-313 Porto, Portugal. Tel.: +351 220428580 fax: +351222003977

$$
\text { E-mail address: Ipeixe@ff.up.pt (L. Peixe) }
$$

${ }^{1}$ These two authors equally contributed to this work.

${ }^{2}$ Present address: University of Bucharest, Faculty of Biology, Department of Microbiology, Portocalelor no. 1-3, district 6, Bucharest, Romania. 
Table 1. Epidemiological and molecular data of carbapenemase-producing Enterobacteriaceae isolated between March-December 2012 from two hospitals in Bucharest, Romania

\begin{tabular}{|c|c|c|c|c|c|c|}
\hline $\begin{array}{l}\text { Species (no. of } \\
\text { isolates) }\end{array}$ & $\begin{array}{l}\text { Hospital } \\
\text { (unit) }^{a}\end{array}$ & Sample (no.) ${ }^{b}$ & $\begin{array}{l}\text { Clone } \\
(\mathrm{ST})^{\mathrm{c}}\end{array}$ & $\begin{array}{l}\text { Carbapenemases and } \\
\text { encoding plasmids (size } \\
\text { in kb) }{ }^{d}\end{array}$ & $\begin{array}{l}\text { ESBL, encoding } \\
\text { plasmid (size in kb) } \\
\text { and additional rep } \\
\text { content }\end{array}$ & $\begin{array}{l}\text { Non- } \beta \text {-lactam } \\
\text { resistance phenotype } \\
\text { e }\end{array}$ \\
\hline $\begin{array}{l}\text { Escherichia coli } \\
\text { (1) }\end{array}$ & $\mathrm{A}(\mathrm{H})$ & Stool (1) & ST5 & NDM-1 $\left(100, F_{Y}\right)$ & CTX-M-15 & $\begin{array}{l}\text { AMK, GEN, TET, } \\
\text { TGC, SXT, CHL, } \\
\text { CST }\end{array}$ \\
\hline $\begin{array}{l}\text { Enterobacter } \\
\text { hormaechei } \\
\text { (6) }\end{array}$ & $A(H, I)$ & $\begin{array}{l}\text { Stool (2), BS (2), } \\
\text { urine (1), PF } \\
(1)\end{array}$ & $\begin{array}{l}\text { ST74, } \\
\text { ST171 }\end{array}$ & $\underline{\text { NDM-1 }}\left(100, F_{Y}\right)$ & CTX-M-15 (290) & $\begin{array}{l}\text { AMK, GEN, NET, } \\
\text { KAN, (TET), (MIN), } \\
\text { (TGC), (SXT), } \\
\text { (CHL), (NIT) }\end{array}$ \\
\hline $\begin{array}{l}\text { K. pneumoniae } \\
\text { (2) }\end{array}$ & $A(U)$ & Urine (1), UN (1) & ST101 & $\begin{array}{l}\text { NDM-1 }{ }^{\mathrm{CR}} ; \text { OXA-181 (60, } \\
\text { L) }\end{array}$ & $\begin{array}{l}\text { CTX-M-15 }(60, \mathrm{~L})+ \\
\mathrm{FII}_{2}+\mathrm{FII}_{\mathrm{K} 1}\end{array}$ & $\begin{array}{l}\text { (AMK), (GEN), NET, } \\
\text { KAN, (TET), (MIN), } \\
\text { (SUL), NIT }\end{array}$ \\
\hline
\end{tabular}




\begin{tabular}{|c|c|c|c|c|c|c|}
\hline $\begin{array}{l}\text { K. pneumoniae } \\
(10)\end{array}$ & $\begin{array}{l}A(I, H) \\
B(I)\end{array}$ & $\begin{array}{l}\text { TS (2), urine (2), } \\
\text { wound (1), } \\
\text { PRF (1), PF } \\
(1), \text { UN (3) }\end{array}$ & ST101 & $\underline{\mathrm{OXA}-48}(60, \mathrm{~L})$ & $\begin{array}{l}\text { CTX-M-15 }(65-80 \\
R)+F I_{K 2}\end{array}$ & $\begin{array}{l}\text { (AMK), (GEN), } \\
\text { (NET), (KAN), } \\
\text { (TET), (MIN), NIT, } \\
\text { (FOF), (CST) }\end{array}$ \\
\hline $\begin{array}{l}\text { K. pneumoniae } \\
\text { (1) }\end{array}$ & $A(H)$ & Stool (1) & ST395 & $\frac{\mathrm{OXA}-48}{15}(50, \mathrm{~L}), \mathrm{CTX}-\mathrm{M}-$ & CTX-M-15 $(75, \mathrm{R})$ & $\begin{array}{c}\text { GEN, NET, TET, } \\
\text { SXT, CHL, NIT }\end{array}$ \\
\hline
\end{tabular}

ESBL, extended-spectrum $\beta$-lactamase.

${ }^{\text {a }}$ A, general hospital; B, cardiovascular hospital; H, haematology; I, intensive care unit; U, urology.

${ }^{b}$ BS, bronchial secretion; PF, pleural fluid; UN, unknown; TS, tracheal secretion; PRF, peritoneal fluid.

${ }^{c}$ Multilocus sequence typing (MLST) was performed on isolates representing different puled-field gel electrophoresis (PFGE) types.

${ }^{\mathrm{d}} \mathrm{CR}$, chromosomal location; transferability of bla genes is shown as underlined.

e AMK, amikacin; GEN, gentamicin; TET, tetracycline; TGC, tigecycline; SXT, trimethoprim/sulfamethoxazole; CHL,

chloramphenicol; CST, colistin; NET, netilmicin; KAN, kanamycin; MIN, minocycline; NIT, nitrofurantoin; SUL, sulphonamides; FOF,

fosfomycin. All isolates were resistant to tobramycin, nalidixic acid and ciprofloxacin. Variability in antibiotic resistance profiles amongst isolates is indicated by parentheses. 